\title{
A 3D model of ovarian cancer cell lines on peptide nanofiber scaffold to explore the cell-scaffold interaction and chemotherapeutic resistance of anticancer drugs
}

\author{
This article was published in the following Dove Press journal: \\ International Journal of Nanomedicine \\ I February 201। \\ Number of times this article has been viewed
}

\author{
Zehong Yang' \\ Xiaojun Zhao',2 \\ 'Nanomedicine Laboratory, West \\ China Hospital and Institute for \\ Nanobiomedical Technology and \\ Membrane Biology, Sichuan University, \\ Chengdu, People's Republic of China; \\ ${ }^{2}$ Center for Biomedical Engineering, \\ Massachusetts Institute of Technology, \\ Cambridge, MA, USA
}

\begin{abstract}
RADA16-I peptide hydrogel, a type of nanofiber scaffold derived from self-assembling peptide RADA16-I, has been extensively applied to regenerative medicine and tissue repair in order to develop novel nanomedicine systems. In this study, using RADA16-I peptide hydrogel, a three-dimensional (3D) cell culture model was fabricated for in vitro culture of three ovarian cancer cell lines. Firstly, the peptide nanofiber scaffold was evaluated by transmission electron microscopy and atom force microscopy. Using phase contrast microscopy, the appearance of the representative ovarian cancer cells encapsulated in RADA16-I peptide hydrogel on days 1, 3, and 7 in 24-well Petri dishes was illustrated. The cancer cell-nanofiber scaffold construct was cultured for 5 days, and the ovarian cancer cells had actively proliferative potential. The precultured ovarian cancer cells exhibited nearly similar adhesion properties and invasion potentials in vitro between RADA16-I peptide nanofiber and type I collagen, which suggested that RADA16-I peptide hydrogel had some similar characteristics to type I collagen. The precultured ovarian cancer cells had two-fold to five-fold higher anticancer drug resistance than the conventional two-dimensional Petri dish culture. So the 3D cell model on peptide nanofiber scaffold is an optimal type of cell pattern for anticancer drug screening and tumor biology.
\end{abstract}

Keywords: 3D culture, anticancer drug, nanofiber scaffold, cell viability, ovarian cancer

\section{Introduction}

Nanotechnology aims to construct novel materials and operative models in nanoscale dimensions. Several potential applications of nanotechnology in medicine can be envisioned, eg, targeted drug delivery systems, tissue engineering scaffolds, novel drug screening strategies, and micro/nanofluidic devices. ${ }^{1}$ Thus far, this field has made progress and merged new ideas. Molecular self-assembly has served as an alternative paradigm for preparing novel nanobiomaterial with a functional nanostructure. Selfassembling peptide was discovered by Shuguang Zhang (Biomedical Engineering Centre, Massachusetts Institute of Technology, MA, USA), who conformed to the 'bottom-up' principles of engineering science. RADA16-I peptide hydrogel was designed to be a matrix scaffold that allows cells to reside in a three-dimensional (3D) microenvironment similar to cellular niches in vivo ${ }^{2}$ and be extensively applied to regenerative medicine and some novel drug delivery systems. ${ }^{3,4}$ The peptide sequence (AcN-RADARADARADARADA- $\mathrm{CONH}_{2}$ ) promoted cell attachment and migration of normal primary somatic cells and some derived stem cells. ${ }^{5,6}$ Recently, some selfassembling peptides were functionalized by functional motifs with a link of two glycine 
residues at the $\mathrm{C}$ terminus. The designer self-assembling peptides enhanced osteoblasts and adult mouse neural stem cell proliferation, differentiation, and 3D migration, which suggests that this novel nanofiber scaffold was a new type of biologically inspired nanomaterial for regenerative medicine and tissue repair. ${ }^{7,8}$ Consequently, self-assembling peptidebased 3D cell culture models are becoming much more useful research tools in modern biomedical investigations, but studies of cancer cell lines grown on this novel nanofiber scaffold, in particular in gynecological oncology, are limited and have provided few insights into tumor biology and anticancer drug screening.

Epithelial ovarian cancer is often an aggressive disease and is typically diagnosed at an advanced stage when therapeutic resistance occurs, for reasons as yet unknown. There are very few studies describing 3D cell culture models for ovarian cancer, which is still poorly understood, and the standard chemotherapeutic regimes are nonspecific. If an elaborate cell culture model that accurately mimics ovarian cancer in vivo can be developed in vitro, the underlying biology of the cancer could be explored and some extensive understanding could be achieved. So the $3 \mathrm{D}$ in vitro cell line models are particularly significant for ovarian cancer. They may be extremely useful tools in gynecological oncology to investigate cellular or molecular events and develop novel anticancer drugs.

In this study, a 3D cell culture model was explored that may provide a type of cell pattern and skills to evaluate the features of peptide nanofiber scaffold and precultured cancer cells. In this article, the ovarian cancer cell lines A2780, A2780/DDP, and SK-OV-3 were seeded in RADA16-I peptide hydrogel for the evaluation of their morphology, adhesion, migration, and chemotherapeutic assay. The 3D cell culture model is an effective tool and preferable for anticancer drug screening and investigations of tumor biology.

\section{Materials and methods Materials}

The peptide RADA16-I, [COCH 3 ]-RADARADARADARADARADA-[CONH ${ }_{2}$ (molecular weight, $1712 \mathrm{kDa}$ ), is commercially available under the name of Puramatrix ${ }^{\mathrm{TM}}$ hydrogel (3DM Inc, Cambridge, MA, USA), which comes packaged as $1 \%(\mathrm{w} / \mathrm{v})$. RADA16-I peptide hydrogel has an approximate $\mathrm{pH}$ value of 4.0 in its packaged state. 3-(4,5-Dimethylthiazol-2-yl)-2,5-diphenyl tetrazolium bromide (MTT) was purchased from Sigma-Aldrich (St Louis, MO, USA). The human ovarian cancer cell lines A2780, A2780/DDP, and SK-OV-3 were purchased from the American Type Culture
Collection (Rockville, MD, USA) and provided by Dr Jinliang Yang at State Key Laboratory of Biotherapy and Cancer Center (Sichuan University, Chengdu, China). To culture ovarian cancer cell lines, penicillin/streptomycin, the media (RPMI-1640, used for A2780 and A2780/DDP; McCoy's 5a Medium, used for SK-OV-3), and fetal bovine serum (FBS) were obtained from Invitrogen (Shanghai, China). Rat-tail type I collagen $(3.56 \mathrm{mg} / \mathrm{mL})$ was purchased from BD Biosciences Inc (San Jose, CA, USA). Anticancer drugs 5-fluorouracil (5-FU), paclitaxel, and curcumin were all purchased from Sigma. All other reagents and solvents were commercially available.

\section{Structural assessment of RADA I6-I peptide nanofiber}

The RADA16-I peptide hydrogel at $1 \%(\mathrm{w} / \mathrm{v})$ was prepared in Milli-Q $\mathrm{H}_{2} \mathrm{O}$ for the transmission electron microscopy as described by $\mathrm{Mi}$ et al. ${ }^{9}$ An aliquot of $1 \mu \mathrm{L}$ was removed and diluted with $19 \mu \mathrm{L}$ of Milli-Q $\mathrm{H}_{2} \mathrm{O}(20 \times$ dilutions). Five microliters of the solution were immediately deposited homogeneously on a freshly cleaved mica surface (about $10 \times 10 \mathrm{~mm}^{2}$ ). Each aliquot was left on the mica for $30 \mathrm{sec}$ and then rinsed with $100 \mu \mathrm{L}$ of Milli-Q $\mathrm{H}_{2} \mathrm{O}$ to remove unattached peptides. The peptide sample on the mica surface was then air dried, and the images were acquired immediately by scanning the mica surface by atom force microscopy (AFM) (Seiko Instruments Inc, Chiba, Japan), operating in tapping mode. Soft silicon cantilevers were chosen, with a length of $219 \mu \mathrm{m}$ and a spring constant of $3 \mathrm{~N} / \mathrm{m}$. The tip radius of curvature was $5 \mathrm{~nm}$. AFM scans were recorded at $512 \times 512$ pixels resolution and produced topographic images in which the brightness of the sample increased as a function of height. Typical scanning parameters have been described. ${ }^{10}$ All image data were subjected to first-order flattening to dispel any distortion, such as reversal of causeless drifting, vibration, and creep of the scanner. AFM observation of each sample was repeated at least three times.

\section{Casting of ovarian cancer cells-seeded hydrogel and 3D cell culture}

For fabricating the ovarian cancer cell-nanofiber scaffold construct, the recommended final concentration of nanofiber scaffold was $0.5 \%(\mathrm{w} / \mathrm{v})$. By using a $1: 1$ dilution of RADA16-I peptide hydrogel to cell suspension in a $20 \%$ sucrose, the final concentrations of nanofiber scaffold and sucrose were $0.5 \%$ and $10 \%$, respectively. The cancer cells of A2780, A2780/DDP, and SK-OV-3 at about $80 \%-85 \%$ confluence were harvested and resuspended in $20 \%$ sucrose 
at the concentration of $1.0 \times 10^{6}$ cells $/ \mathrm{mL}$. The cancer cell suspension was quickly mixed with an equal volume of RADA16-I peptide hydrogel, and $50 \mu \mathrm{L}$ of the mixture was quickly loaded into the top portion of each well in a 24-well Petri dish ( $100 \mu \mathrm{L}$ for 12 -well Petri dish) before $250 \mu \mathrm{L}$ of the culture medium was immediately added to the bottom portion of each well ( $500 \mu \mathrm{L}$ for a 12 -well Petri dish). The seeding density was fixed at a viable cell concentration of $1.0 \times 10^{6}$ cells $/ \mathrm{cm}^{2}$. The addition of a cell medium caused RADA16-I peptide hydrogel to self-assemble or gel in about 30-60 min and encapsulate cancer cells in a $3 \mathrm{D}$ microenvironment. The media were changed twice in the next $30 \mathrm{~min}$, and care was taken not to aspirate the top portion of the Petri dish. The procedure of $3 \mathrm{D}$ cell culture was performed over 7 days, and the media were replaced every other day.

To assess the cell proliferation in $3 \mathrm{D}$ cell culture, the cell viability was analyzed by the assay at the desired time. All cell cultures were made in the complete medium with $10 \% \mathrm{FBS}$ and antibiotics in a humidified atmosphere under $5 \% \mathrm{CO}_{2}$ at $37^{\circ} \mathrm{C}$. The cancer cells $\left(5 \times 10^{4}\right.$ cells/well $)$ were seeded on the nanofiber scaffold in a 24 -well Petri dish. The cell proliferation was monitored by an MTT assay for 1, 3, 5, and 7 days with a microplate reader (Bio-Rad Inc, Hercules, CA, USA). The mechanism of this assay is that biologically active cells react with tetrazolium salt in the MTT reagent to produce water-insoluble formazan dye that can be observed at $570 \mathrm{~nm}$.

\section{Adhesion assay}

The adhesion assays of the ovarian cancer cells on the peptide nanofiber scaffold and type I collagen scaffold $(1.78 \mathrm{mg} / \mathrm{mL})$ were performed as previously described with some modifications. ${ }^{11}$ Ninety-six-well flat-bottom poly(vinyl chloride) (PVC) titer plates were coated with $50 \mu \mathrm{L}$ of RADA16-I peptide hydrogel and type I collagen, following incubation overnight at $4^{\circ} \mathrm{C}$. The self-assembly process of peptide nanofiber was initiated by adding $50 \mu \mathrm{L}$ of phosphate-buffered saline (PBS) to the plate. The hydrogel solution was then removed and the wells were washed twice with PBS and incubated with $10 \mathrm{mg} / \mathrm{mL}$ bovine serum albumin (BSA) in PBS for 30 min to block the uncoated PVC surface. The wells were rinsed twice with PBS before the addition of cells. For the adhesion assay, the ovarian cancer cells were collected for use after being cultured for 5 days in a $3 \mathrm{D}$ cell culture model. The cancer cell layer was rinsed with the ice-cold PBS and scraped into PBS on ice and counted in a hemacytometer. The cell suspension was diluted to obtain a concentration of $1.0 \times 10^{5}$ cells $/ \mathrm{mL}$.
About $50 \mu \mathrm{L}$ of cell suspension was added to each well and incubated at $37^{\circ} \mathrm{C}$ for $4 \mathrm{~h}$, and then the cell suspension was removed from the wells, which were rinsed twice with PBS. The cancer cells in the well were measured by an MTT assay. Adhesion assay of each ovarian cancer cell line was carried out at least in triplicate.

\section{Migration and invasion assay}

In order to explore the migration and invasion potential of ovarian cancer cells on the peptide nanofiber scaffold, a model of tumor cell migration and invasion was established on the basis of the conventional transwell model as introduced previously. ${ }^{12-14}$ Millicell-polycarbonate (PCF) insert (Millipore, Billerica, MA, USA) was used for the cell migration and invasion assay. The chamber consisted of a 24-well plate and Millicell insert, a transparent PCF membrane with $8-\mu \mathrm{m}$ pore size. First, the millicell inserts were coated with a thin layer of RADA16-I peptide hydrogel or type I collagen solution. The precultured cancer cells of A2780, A2780/DDP, and SK-OV-3 were resuspended in the starvation medium, the complete medium containing $1 \% \mathrm{FBS}$, to prepare the condition medium for use. Ovarian cancer cells $\left(1 \times 10^{5}\right.$ cells $\left./ \mathrm{mL}\right)$ of $100 \mu \mathrm{L}$ were added to the inserts and incubated at $37^{\circ} \mathrm{C}$ for $24 \mathrm{~h}$. The cancer cells adhered to the upper side of the membrane were removed gently with a cotton swab and rinsed with PBS. The cells that migrated through the membrane and adhered to the bottom of the membrane were fixed in 4\% paraformaldehyde and stained with $0.1 \%$ crystal violet. The PCF membrane was cut out and photographed. This assay was performed in triplicate for every ovarian cancer cell line.

\section{Chemotherapeutic assay}

In order to evaluate the chemotherapeutic effect of the anticancer drug in a $3 \mathrm{D}$ cell culture model, the $\mathrm{IC}_{50}$ value, which was the mean of the concentration of anticancer drug achieving a $50 \%$ inhibition of cell viability as compared with the untreated cell control, was firstly determined in the conventional two-dimensional (2D) Petri dish. 5-FU, paclitaxel, and curcumin were dissolved in dimethyl sulfoxide at $100 \mathrm{mM}$ as stock solutions and diluted to the proper concentration with culture medium. A2780, A2780/DDP, and SK-OV-3 cells were cultured in a 24-well Petri dish at a cell density of $5 \times 10^{4}$ cells/well for $24 \mathrm{~h}$ in the complete media. For drug treatment, the culture medium was renewed with a fresh medium containing the proper concentration of the anticancer drug, and then the cancer cells were incubated for another $24 \mathrm{~h}$. The cell viability was determined by MTT assay. 
The ovarian cancer cells of A2780, A2780/DDP, and SKOV-3 were seeded on the peptide nanofiber scaffold in a 12-well Petri dish and cultured for 5 days in the media before adding the anticancer drugs. The culture medium was renewed with the fresh medium containing two-fold to five-fold $\mathrm{IC}_{50}$ concentration of the anticancer drug. Chemotherapeutic effect was evaluated by an MTT assay after another 24-h incubation.

\section{Statistics}

All data were expressed as mean \pm standard deviation and an ANONA and Student's $t$-test were conducted with SPSS version 13.0 software (SPSS, Chicago, IL, USA). Results for all analyses with a $P$ value $<0.05$ indicated the statistically significant differences $(* P<0.05 ; * * P<0.01)$.

\section{Results and discussion Molecular characterization of RADA I6-I peptide nanofiber scaffold}

Molecular self-assembling peptide RADA16-I derived from a Lego peptide segment of Zuotin, a left-handed Z-DNA-binding protein originally discovered in yeast, with alternating alanine and amino acid residues, has recently emerged as a novel nanobiomaterial for regenerative medicine and tissue repair. ${ }^{15-17}$ RADA16-I peptide served as the building block of nanofiber scaffold $\approx 10 \mathrm{~nm}$ in diameter with the drive of positively and negatively charged residues by complementary ionic interactions and spontaneously assembled into the well-ordered nanofiber networks with the pore size of about 5-200 nm (Figure 1A and B). When dissolved in Milli-Q $\mathrm{H}_{2} \mathrm{O}$, RADA16-I peptide existed as the stable hydrogel with extremely high water content $(>99.5 \%$ ) at $1-5 \mathrm{mg} / \mathrm{mL}(\mathrm{w} / \mathrm{v})$. The in situ AFM scan exhibited that RADA16-I peptide nanofiber scaffold consisted of the nanofiber with length $1019.83 \pm 90.16 \mathrm{~nm}$ and diameter $32.37 \pm 1.72 \mathrm{~nm}$ (Figure 1C and D), which closely mimicked the porosity and gross structure of extracellular matrices (ECMs) and was suitable for the fabrication of the artificial cell niches for the applications of tumor biology and regenerative medicine. When desired, the cancer cells could be easily encapsulated into the nanofiber networks in a truly $3 \mathrm{D}$ microenvironment. When mixed with the ovarian cancer cells, the peptide nanofiber hydrogel formed the regular cancer cell-nanofiber scaffold construct, which was readily mounted on the bottom of the wells in a Petri dish. The cancer cells were evenly distributed and enabled the user to develop the different cell patterns in line with the particular purpose of cell biology. The operation procedure was very simple and convenient in the laboratory.

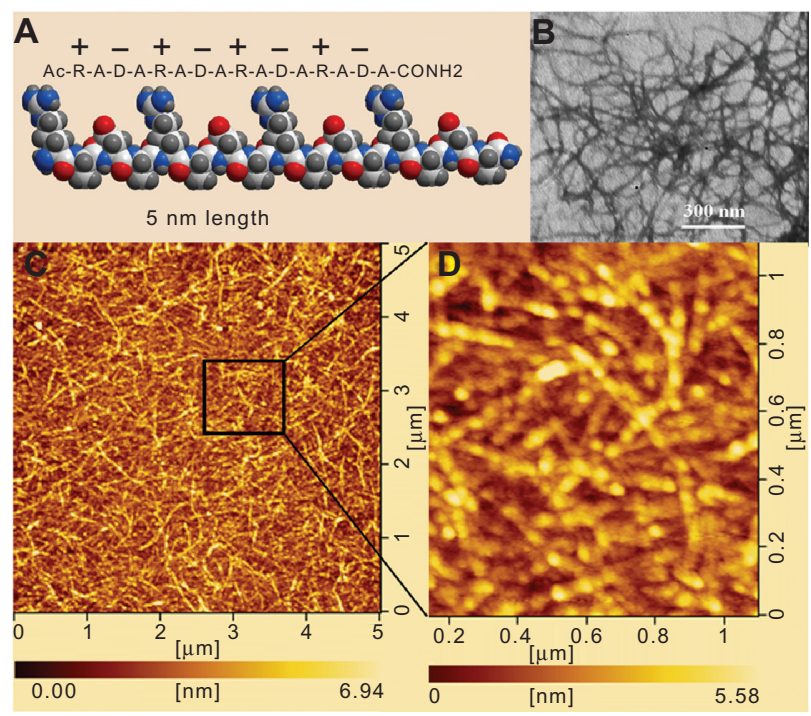

Figure I Molecular self-assembling peptide RADA I6-I was evaluated by TEM and AFM. A) Molecular model of RADAI6-I with 16 amino acid residues and the alternating positive and negative residue's arrangement. B) TEM of RADAI6-I peptide nanofiber (magnified $\times 40,000$ ). C) The interwoven nanofiber networks of RADA I6-I peptide by AFM scan. D) High-resolution deflection image of RADA I6-I peptide nanofiber. The nanofiber scaffold presented here was observed at least three times.

Abbreviations: TEM, transmission electron microscopy; AFM, atom force microscopy.

\section{Morphology and proliferation of ovarian cancer cells}

As 3D cell models for ovarian cancer, A2780, A2780/DDP, and SK-OV-3 cell lines were chosen; the A2780 cells had nearly the same features as A2780/DDP, besides the different drug resistance. SK-OV-3 cells had slow growth activity. The ovarian cancer cell-peptide nanofiber construct cast in the 24-well Petri dish was of a particular transparent morphology that enabled the convenient capture of cell images by the phase contrast microscopy. The cancer cell-peptide nanofiber scaffold interactions were intimate and clear-cut in appearance. The peptide nanofibers were densely enmeshed with each other, and the cancer cells gradually integrated with the surrounding nanofibers as cell growth proceeded. On the first day, the cancer cells of A2780, A2780/DDP, and SK-OV-3 were evenly distributed and maintained inherent morphology (Figure 2A, D, and G). After 3 days, cell density increased; simultaneously, the cells closed up and presented distinctive appearances. A2780 and A2780/DDP cells initially formed cell colonies, whereas SK-OV-3 cells proliferated slowly and maintained their individual cellular appearance (Figure 2B, E, and H). After 7 days, A2780 and A2780/DDP cells formed the multicellular spheroids (Figure 2C and F), whereas SK-OV-3 cells formed the cell clusters (Figure 2I), which were the typical morphologies of cancer cells in 3D cell culture and the common 

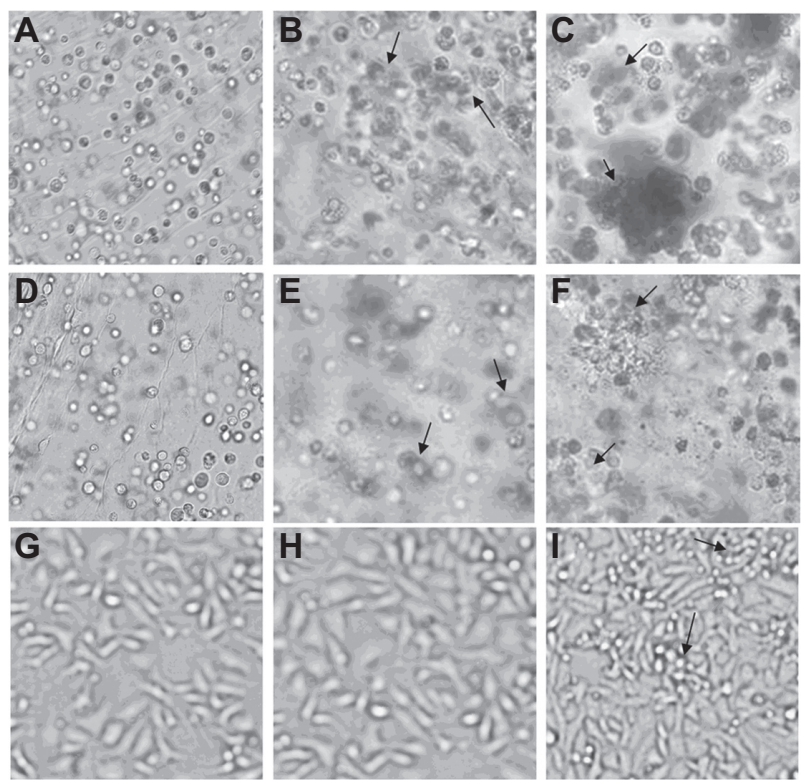

Figure 2 Phase contrast microscopy images of A-C) A2780, D-F) A2780/DDP, and G-I) SK-OV-3 cultured in RADA I6-I peptide hydrogel on days I, 3, and 7. The arrows indicate multicellular spheroids, cell clusters, or cell colonies. A, D, and $\mathbf{G}$ ) indicate the initial cell morphology on the first day. $\mathbf{B}, \mathbf{E}$, and $\mathbf{H}$ ) indicate the cell colonies of A2780, A2780/DDP, and SK-OV-3 after 3 days. C, F, and I) indicate the multicellular spheroids of A2780 and A2780/DDP and the cell clusters of SK-OV-3 after 7 days. All images are captured at $\times 200$ magnification.

characteristics of the glandular epithelial tumors. As 3D cell culture continued, the cell morphologies of A2780, A2780/DDP, and SK-OV-3 cells maintained the inherent characteristics, and SK-OV-3 cells did not form the multicellular spheroids. The more detailed morphologies of A2780, A2780/DDP, and SKOV-3 cells were difficult to observe in the laboratory. The use of RADA16-I peptide hydrogel to culture cancer cells should improve our understanding of the disease and allow us to assess treatment response in more clinically predictive model systems. ${ }^{18}$ RADA16-I peptide hydrogel conferred an elaborate 3D microenvironment for cancer cells in response to the surrounding topography. The 3D cell culture model was a simple cell encapsulating methodology and an optimal strategy for tumor biology and regenerative medicine.

\section{Cancer cell viability}

MTT assay was performed to assess the cell viability of three ovarian cancer cell lines on a peptide nanofiber scaffold. In a 3D cell culture procedure, very few cancer cells underwent apoptosis and necrosis. As Figure 3 indicates, the cell proliferation of three ovarian cancer cell lines exhibited similar cell viability in a 3D cell culture model, although SK-OV-3 cells proliferated slowly. When cultured for 5 days, all three ovarian cancer lines showed the significantly higher proliferative activity, indicating that ovarian cancer cells acclimatized themselves to the 3D

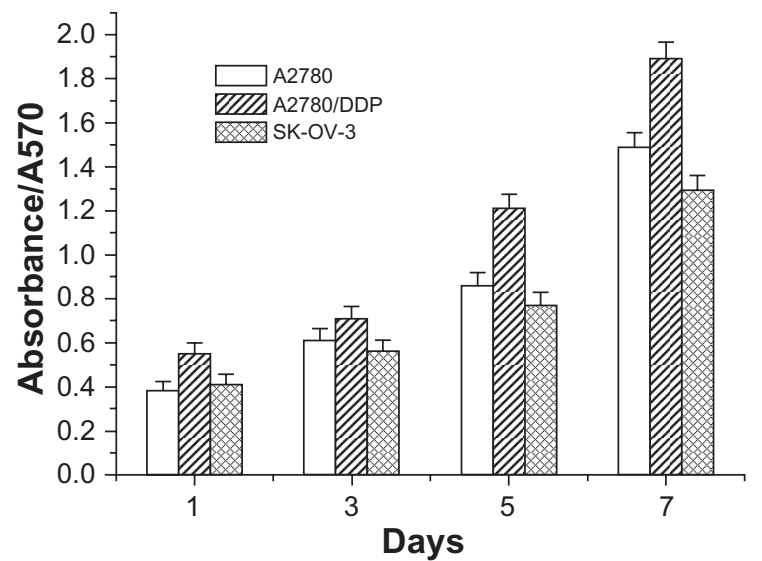

Figure 3 Cell viability of three ovarian cancer cell lines as a function of culture time. $5 \times 10^{4}$ cells were seeded on the peptide nanofiber scaffold and monitored by an MTT assay with a microplate reader.

Abbreviation: MTT, 3-(4,5-dimethylthiazol-2-yl)-2, 5-diphenyl tetrazolium bromide.

microenvironment of a peptide nanofiber scaffold. It is unanimously recognized that the in vivo microenvironment of cancer cells is key to metastasis and tumor progression. The peptide nanofiber hydrogel formed the elaborate 3D microenvironment that was similar to the tumor in vivo and facilitated the maintenance of the inherent malignant phenotype of the ovarian cancer cells. Recent progress in bioengineered tumor models has revealed that the nanofiber scaffold is suitable for cancer cell growth and survival. The nanofiber scaffold potentially provides some functionalities for cell proliferation, adhesion, migration, invasion, and phenotype presentations. ${ }^{19,20}$ The cell morphology and cancer cell-nanofiber scaffold construct were studied for 7 days. When cultured in the 3D model for 5 days, the ovarian cancer cells exhibited actively proliferative potential, which encourages investigation in tumor biology and may lead to the discovery of anticancer drugs.

\section{Adhesion of ovarian cancer cells on the peptide nanofiber}

The cell-matrices interaction was an important determinant of cancer cell behavior and tumorigenesis. Type I collagen was a structural protein in the ECMs and was widely used as a scaffold and promoter for cell adhesion. The natural fibrous structure of type I collagen could promote the cells to attach to the interstitial matrix. ${ }^{21}$ The precultured ovarian cancer cells of A2780, A2780/DDP, and SK-OV-3 all showed significant attachment potential to type I collagen and the peptide nanofiber scaffold as compared with the control BSA. No significant adhesion difference occurred between type I collagen and the peptide nanofiber scaffold (Figure 4A). The adhesion percentages of type I collagen and the peptide nanofiber scaffold also had no significant 

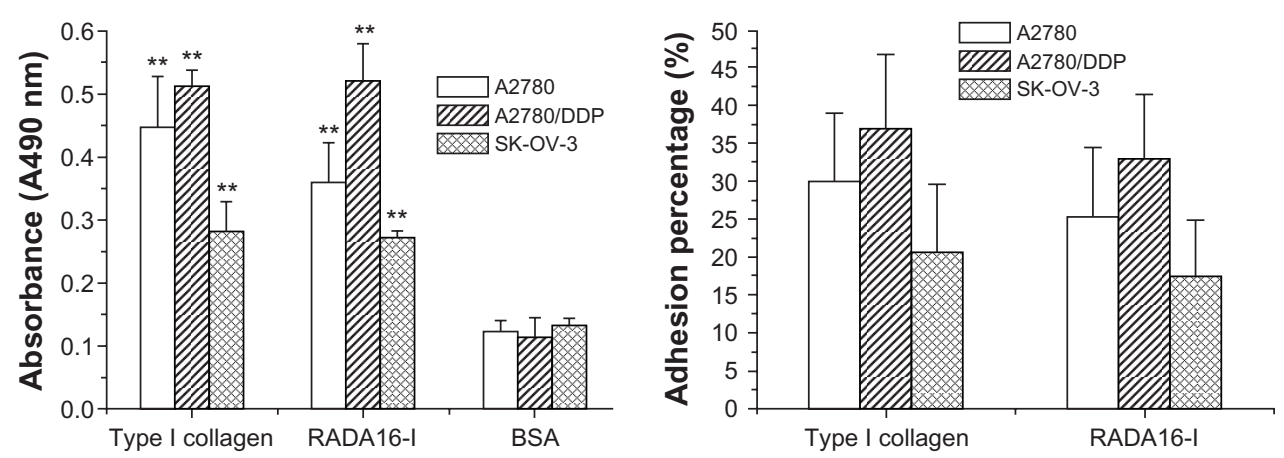

Figure 4 Adhesion behavior of the precultured A2780, A2780/DDP, and SK-OV-3 cells. A) The number of adhesion cells on the type I collagen, the peptide nanofiber, and BSA (control). B) The adhesion percentage of the precultured A2780, A2780/DDP, and SK-OV-3 cells on the scaffolds. The assay was conducted at least in triplicate. Abbreviation: BSA, bovine serum albumin.

statistical differences (Figure 4B), which suggested that self-assembling peptide RADA16-I nanofiber scaffold had some similar characteristics of type I collagen. Thus far, in the case of neural stem cells, RADA16-I peptide nanofiber scaffold has comprehensively been compared with the most commonly used scaffolds, including PLLA, PLGA, PCLA, type I collagen, type IV collagen, and Matrigel, which indicates that RADA16-I peptide nanofiber scaffold represents a new class of functionalized and biologically active nanofiber scaffold. ${ }^{22}$ The well-defined nanofiber networks and the characteristics of the high $\mathrm{H}_{2} \mathrm{O}$ content make it a very attractive class of biological nanomaterial. It is favorable for cell adhesion and differentiation. ${ }^{23}$ Broadly, these studies are consistent with our data. So the 3D cell model developed in this study was a good representation of the primary tumor and likely to simulate the in vivo biological characteristics of ovarian cancer cells. ${ }^{24}$

\section{Migration and invasion of the ovarian cancer cells}

When ovarian cancer cells were able to adhere to the peptide nanofiber scaffold, whether these ovarian cancer cells were able to migrate or infiltrate the nanofiber matrices in the 3D model was investigated. As Figure 5 indicates, three ovarian cancer cell lines readily adhered to the peptide nanofiber scaffold and invaded the gelled peptide hydrogel matrices after 24-h culture. There was no significant difference between the number of migrating cancer cells for type I collagen and the peptide nanofiber scaffold, which suggests that the peptide nanofiber scaffold had some biological functionalities that were similar to type I collagen and facilitated the maintenance of the malignant phenotypes of ovarian cancer cells or the invasion potentials. Although the molecular mechanisms of the cancer cells' adhesion and invasion were delicate in both normal and pathological conditions, in the case of highly aggressive ovarian cancer cells, it was imperative that the basic molecular or cellular events remained to be elucidated in a defined cell culture model. So the 3D cell culture model in this study offered a type of cell pattern to explore the topographical features at nanoscale comparable with the scale of the ECM components and the biological activities of tumorigenesis, metastasis, and colonization. 16,17,23,25,26

\section{Chemotherapeutic assay of anticancer drugs}

In the anticancer drug screening laboratory, the $2 \mathrm{D}$ cell culture was often used to monitor the cytostatic effects of the different anticancer drugs; the malignant cells were only able to obtain cell viability and increase the cell number. In contrast, a 3D cell culture model fabricated on the nanofiber scaffold conferred on the precultured cancer cells some cellular functionalities to enable them to survive in the

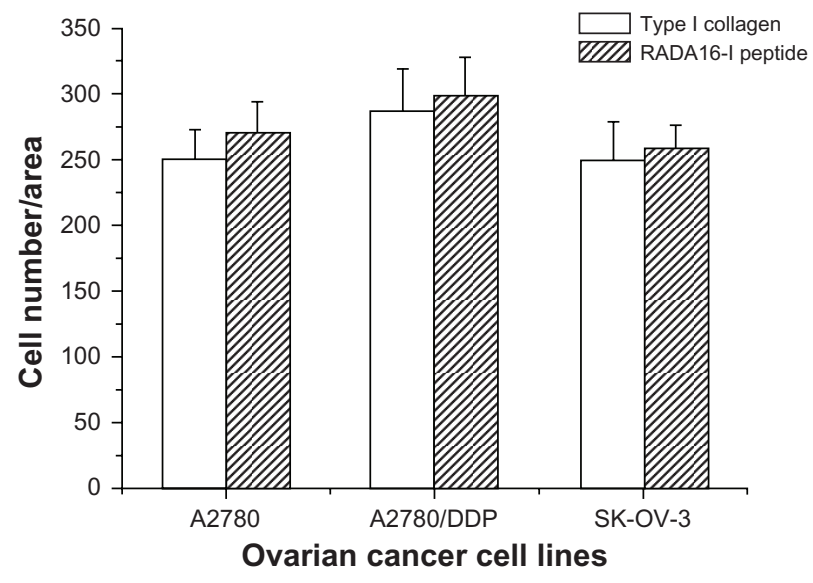

Figure 5 The migration and invasion of the precultured A2780, A2780/DDP, and SK-OV-3 cells. Relative normalized cell number per area was counted under the condition of phase contrast microscopy. This assay was performed in triplicate for every ovarian cancer cell line. 
Table I $I C_{50}$ concentration in the 2D Petri dish culture and chemotherapeutic assay in the $3 \mathrm{D}$ model

\begin{tabular}{llll}
\hline $\begin{array}{l}\text { Anticancer } \\
\text { drug }\end{array}$ & $\begin{array}{l}\text { Tested concentration } \\
(\mu \mathrm{g} / \mathrm{mL})\end{array}$ & $\mathrm{IC}_{50}(\mu \mathrm{g} / \mathrm{mL})$ & $\begin{array}{l}\text { 3D model } \\
(\mu \mathrm{g} / \mathrm{mL})\end{array}$ \\
\hline 5-FU & $\mathrm{I}-500$ & 120 & 350 \\
Paclitaxel & $\mathrm{I}-500$ & 90 & 370 \\
Curcumin & $\mathrm{I}-300$ & 35 & 80 \\
\hline
\end{tabular}

Note: The concentration values were determined using $5 \times 10^{4}$ cell number.

Abbreviations: 2D, two-dimensional; 3D, three-dimensional; 5-FU, 5-fluorouracil; $\mathrm{IC}_{50}$, mean of the concentration of anticancer drug achieving a 50\% inhibition of cell viability.

in vivo microenvironment and profoundly affect the response of the ovarian cancer cells to chemotherapeutic drugs. The precultured ovarian cancer cells exhibited increased resistance compared with the cancer cells in 2D Petri dish culture (Table 1). Initially, the $\mathrm{IC}_{50}$ concentrations of 5-FU, paclitaxel, and curcumin were determined. The precultured ovarian cancer cells needed two-fold to five-fold higher concentrations of the anticancer drugs to reach more than $50 \%$ inhibition activity, as described in Figure 6, indicating that the chemotherapeutic resistance was derived from the inherent properties of the precultured ovarian cancer cells. The 3D cell culture model may have provided a cancerous microenvironment that was more reflective of the tumor in vivo. ${ }^{27}$ Tumorigenesis was related to the cancer cell-matrices microenvironment, which determined how aggressive the tumor might be and how to respond to the chemotherapeutic intervention. ${ }^{24}$ The cell culture operations on the peptide nanofiber scaffold are simple and easy to set up, and the maintenance of cell lines is also simple. So the 3D cell model may be used in technological

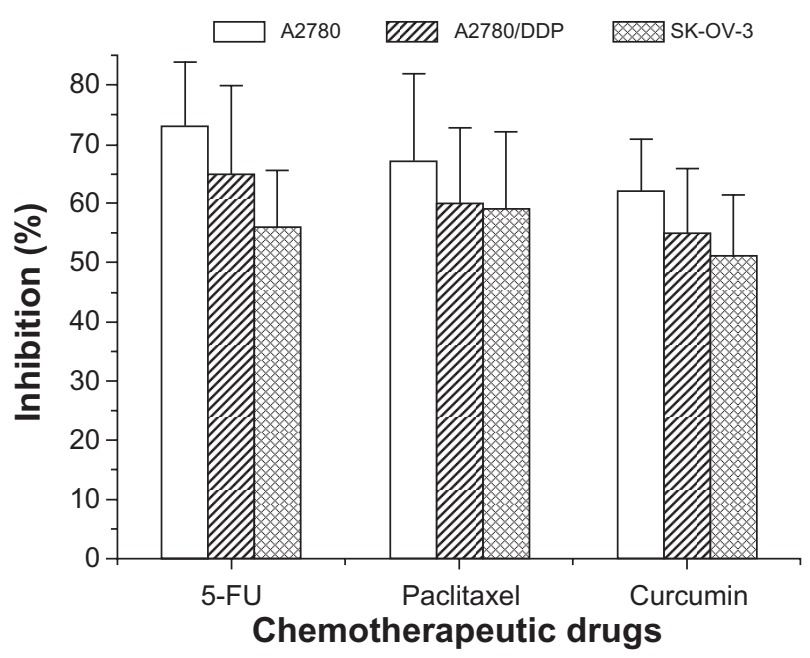

Figure 6 Chemotherapeutic effects of the anticancer drugs against the precultured ovarian cancer cells. Cancer cells in the conventional two-dimensional Petri dish and the precultured cancer cells in peptide nanofiber scaffold were evaluated by the cell viability after 24-h incubation.

Abbreviation: 5-FU, 5-fluorouracil. development for anticancer drug screening and is particularly useful in guiding the treatment regimes for untraceable cancerous diseases with high mortality. Other studies are underway in our laboratory to explore the molecular markers specifically relevant to the progression of epithelial ovarian cancer cells grown in $3 \mathrm{D}$ cell patterns and to test the anticancer drugs in orthotopic mouse models.

\section{Conclusion}

In this study, the molecular self-assembling peptide RADA16-I forms a well-ordered nanofiber network and stable hydrogel. The cell casting procedure is simple and enables a rapid and efficient encapsulation of ovarian cancer cells within RADA16-I peptide hydrogel. Encapsulated cells are evenly distributed throughout the hydrogel. The ovarian cancer cells exhibit actively proliferative activities and maintain the inherent characteristics of cell morphology. Furthermore, compared with type I collagen, the most common ECM, RADA16-I peptide nanofiber, is preferable for ovarian cancer cell lines to adhere and migrate in a 3D environment. Interestingly, compared with the conventional 2D Petri dish culture, the precultured ovarian cancer cells exhibit obviously higher therapeutic resistance to anticancer drugs, including 5-FU, paclitaxel, and curcumin. So a 3D cell culture model in RADA16-I peptide hydrogel can be particularly useful for anticancer drug screening and in vitro investigation of tumor biology. RADA16-I peptide hydrogel is a significant advance in nanobiomaterial innovation and provides an effective type of cell pattern for regenerative medicine and tumor biology.

\section{Acknowledgments}

The authors are grateful to Dr Honggang Lin and Dr Jinliang Yang from State Key Laboratory of Biotherapy and Cancer Center (Sichuan University, Chengdu, China) for their suggestions and assistance with this work. This study has been supported by ' 985 engineering' of Sichuan University, Chengdu, China.

\section{Disclosure}

The authors report no conflicts of interest in this work.

\section{References}

1. Rajagopal K, Schneider JP. Self-assembling peptides and proteins for nanotechnological applications. Curr Opin Struct Biol. 2004;14(4):480-486.

2. Zhang S, Gelain F, Zhao X. Designer self-assembling peptide nanofiber scaffolds for 3D tissue cell cultures. Semin Cancer Biol. 2005; 15(5):413-420.

3. Zhang S. Designer self-assembling peptide nanofiber scaffolds for study of 3-d cell biology and beyond. Adv Cancer Res. 2008;99:335-362. 
4. Zhang S. Fabrication of novel biomaterials through molecular selfassembly. Nat Biotechnol. 2003;21(10):1171-1178.

5. Semino CE, Kasahara J, Hayashi Y, Zhang S. Entrapment of migrating hippocampal neural cells in three-dimensional peptide nanofiber scaffold. Tissue Eng. 2004;10(3-4):643-655.

6. Zhang S, Holmes TC, DiPersio CM, Hynes RO, Su X, Rich A. Self-complementary oligopeptide matrices support mammalian cell attachment. Biomaterials. 1995;16(18):1385-1393.

7. Horii A, Wang X, Gelain F, Zhang S. Biological designer selfassembling peptide nanofiber scaffolds significantly enhance osteoblast proliferation, differentiation and 3-D migration. PLoS One. 2007; 2(2): 190 .

8. Gelain F, Bottai D, Vescovi A, Zhang S. Designer self-assembling peptide nanofiber scaffolds for adult mouse neural stem cell 3-dimensional cultures. PLoS One. 2006;1:e119.

9. Mi K, Wang G, Liu Z, Feng Z, Huang B, Zhao X. Influence of a selfassembling peptide, RADA16, compared with collagen I and matrigel on the malignant phenotype of human breast-cancer cells in 3D cultures and in vivo. Macromol Biosci. 2009;9(5):437-443.

10. Ye Z, Zhang $\mathrm{H}$, Luo $\mathrm{H}$, et al. Temperature and $\mathrm{pH}$ effects on biophysical and morphological properties of self-assembling peptide RADA16-I. J Pept Sci. 2008;14(2):152-162.

11. Kokenyesi R, Murray KP, Benshushan A, Huntley ED, Kao MS. Invasion of interstitial matrix by a novel cell line from primary peritoneal carcinosarcoma, and by established ovarian carcinoma cell lines: role of cell-matrix adhesion molecules, proteinases, and E-cadherin expression. Gynecol Oncol. 2003;89(1):60-72.

12. Zhang S, Lockshin C, Herbert A, Winter E, Rich A. Zuotin, a putative Z-DNA binding protein in Saccharomyces cerevisiae. EMBO J. 1992; 11(10):3787-3796.

13. Valster A, Tran NL, Nakada M, Berens ME, Chan AY, Symons M. Cell migration and invasion assays. Methods. 2005;37(2):208-215.

14. Maliakal JC. Quantitative high throughput endothelial cell migration and invasion assay system. Methods Enzymol. 2002;352:175-182.

15. Zhang S, Marini DM, Hwang W, Santoso S. Design of nanostructured biological materials through self-assembly of peptides and proteins. Curr Opin Chem Biol. 2002;6(6):865-871.
16. Zhang S, Yan L, Altman M, et al. Biological surface engineering: a simple system for cell pattern formation. Biomaterials. 1999;20(13): 1213-1220.

17. Gelain F, Lomander A, Vescovi AL, Zhang S. Systematic studies of a self-assembling peptide nanofiber scaffold with other scaffolds. J Nanosci Nanotechnol. 2007;7(2):424-434.

18. Abu-Yousif AO, Rizvi I, Evans CL, Celli JP, Hasan T. PuraMatrix encapsulation of cancer cells. J Vis Exp. 2009;34(Pt 2):1692. Doi:10.3791/1692.

19. Loessner D, Stok KS, Lutolf MP, Hutmacher DW, Clements JA, Rizzi SC. Bioengineered 3D platform to explore cell-ECM interactions and drug resistance of epithelial ovarian cancer cells. Biomaterials. 2010; 31(32):8494-8506.

20. Fischbach C, Chen R, Matsumoto T, et al. Engineering tumors with 3D scaffolds. Nat Methods. 2007;4(10):855-860.

21. Provenzano PP, Eliceiri KW, Inman DR, Keely PJ. Engineering threedimensional collagen matrices to provide contact guidance during $3 \mathrm{D}$ cell migration. Curr Protoc Cell Biol. 2010; Chapter 10:Unit 10.17.

22. Naskar J, Palui G, Banerjee A. Tetrapeptide-based hydrogels: for encapsulation and slow release of an anticancer drug at physiological $\mathrm{pH}$. J Phys Chem B. 2009;113(35):11787-11792.

23. Melani M, Simpson KJ, Brugge JS, Montell D. Regulation of cell adhesion and collective cell migration by hindsight and its human homolog RREB1. Curr Biol. 2008;18(7):532-537.

24. Grun B, Benjamin E, Sinclair J, et al. Three-dimensional in vitro cell biology models of ovarian and endometrial cancer. Cell Prolif. 2009; 42(2):219-228

25. Heydarkhan-Hagvall S, Choi CH, Dunn J, et al. Influence of systematically varied nano-scale topography on cell morphology and adhesion. Cell Commun Adhes. 2007;14(5):181-194.

26. Kim YJ, Bae HI, Kwon OK, Choi MS. Three-dimensional gastric cancer cell culture using nanofiber scaffold for chemosensitivity test. Int J Biol Macromol. 2009;45(1):65-71.

27. Horning JL, Sahoo SK, Vijayaraghavalu S, et al. 3-D tumor model for in vitro evaluation of anticancer drugs. Mol Pharm. 2008;5(5):849-862.
International Journal of Nanomedicine

\section{Publish your work in this journal}

The International Journal of Nanomedicine is an international, peerreviewed journal focusing on the application of nanotechnology in diagnostics, therapeutics, and drug delivery systems throughout the biomedical field. This journal is indexed on PubMed Central, MedLine, CAS, SciSearch ${ }^{\circledR}$, Current Contents ${ }^{\circledR} /$ Clinical Medicine,

\section{Dovepress}

Journal Citation Reports/Science Edition, EMBase, Scopus and the Elsevier Bibliographic databases. The manuscript management system is completely online and includes a very quick and fair peer-review system, which is all easy to use. Visit http://www.dovepress.com/ testimonials.php to read real quotes from published authors. 